\title{
COMMUNICATIONS
}

\section{The dynamic behavior of butanethiol and dodecanethiol adsorbates on Au(111) terraces}

\author{
F. Terán Arce, M. E. Vela, R. C. Salvarezza, and A. J. Arvia ${ }^{a)}$ \\ Instituto de Investigaciones Fisicoquímicas Teóricas y Aplicadas (INIFTA), Sucursal 4, Casilla de Correo \\ 16. (1900) La Plata, Argentina
}

(Received 30 April 1998; accepted 30 July 1998)

\begin{abstract}
The dynamics of butanethiol and dodecanethiol monolayers adsorbed on $\mathrm{Au}(111)$ studied by ex situ and in situ sequential scanning tunneling microscopy (STM) shows, at room temperature and in the range of seconds, $\sqrt{ } 3 \times \sqrt{ } 3 R 30^{\circ} \Leftrightarrow c(4 \times 2)$ transitions. High-resolution STM imaging also shows that these transitions can be explained by a displacement of adsorbed molecules from hollow to bridge sites and vice versa. Transitions from the $p(n \times 1)$ superlattice to the $\sqrt{ } 3 \times \sqrt{ } 3 R 30^{\circ}$ lattice were also imaged in real time. These processes appear to be coupled with fluctuations of the hole size of the $\mathrm{Au}(111)$ terrace. (C) 1998 American Institute of Physics. [S0021-9606(98)70838-4]
\end{abstract}

In recent years self-assembled monolayers (SAMs) formed when methyl-terminated thiol chains are chemisorbed on different substrates have been extensively studied, particularly on $\mathrm{Au}(111)$, in relation to different processes such as molecular assembly, wetting, tribology, and corrosion inhibition. ${ }^{1-7}$ These adsorbed layers exhibit either a $\sqrt{ } 3$ $\times \sqrt{ } 3 R 30^{\circ}$ hexagonal lattice or a $c(4 \times 2)$ rectangular superlattice, which have been observed by different techniques, such as grazing incidence $\mathrm{x}$-ray diffraction, ${ }^{8,9} \mathrm{He}$ diffraction, ${ }^{10}$ and scanning tunneling microscopy (STM). ${ }^{9}$ STM imaging data have shown that domains with the $\sqrt{3}$ $\times \sqrt{ } 3 R 30^{\circ}$ lattice and the $c(4 \times 2)$ superlattice coexist with monatomic deep holes on the $\mathrm{Au}(111)$ surface, irrespective of the thiol-chain length and SAM preparation.

Two different models ( $I$ and $I I$ ) have been proposed to explain the $c(4 \times 2)$ structure. Model $I^{9}$ states that the $c(4$ $\times 2)$ superlattice results from thiol molecules with different twist angles forming rows with different contrast in the STM image. Otherwise, model $I^{11}$ admits that each head in the $\sqrt{ } 3 \times \sqrt{ } 3 R 30^{\circ}$ lattice results from a S-S bond, while the terminal hydrocarbon chain axis remains with a nearly $\sqrt{ } 3$ $\times \sqrt{ } R 30^{\circ}$ hexagonal two-dimensional packing. This arrangement produces a gauche $\mathrm{S}-\mathrm{C}$ bond with a $\sim 0.2 \mathrm{~nm}$ distance between the $\mathrm{S}$ head groups and the hydrocarbon chain axis, and a $0.22 \mathrm{~nm} \mathrm{~S}-\mathrm{S}$ distance. Therefore, model II states that the equilibrium structure of the adsorbed layer is in the form of a disulfide. This conclusion from model $I I$, however, contradicts the fact that the $\mathrm{S}-\mathrm{S}$ bond in disulfide molecules adsorbed on $\mathrm{Au}(111)$ is unstable, being cleaved during annealing to $100{ }^{\circ} \mathrm{C}^{12}$ Conversely, it has also been reported that the $\sqrt{ } 3 \times \sqrt{ } 3 R 30^{\circ}$ structure changes into the $c(4 \times 2)$ superlattice after either annealing or successive scanning of the thiol layer with the STM tip. ${ }^{13}$ Accordingly, it has been concluded that the $c(4 \times 2)$ superlattice is more

\footnotetext{
${ }^{a)}$ Present address: Instituto de Investigaciones Fisicoquímicas, Teóricas y Aplicadas (INIFTA), Casilla de Correo 16, Sucursal 4, (1900) La Plata, Argentina. Fax: 54-21-254642; Electronic mail: ajarvia@isis.unlp.edu.ar
}

stable than the $\sqrt{ } 3 \times \sqrt{ } 3 R 30^{\circ}$ lattice. ${ }^{13}$ From the above, it can be concluded that the origin of the different surface structures for thiol adsorbates on $\mathrm{Au}(111)$ still remains unclear.

In this communication we report ex situ and in situ STM sequential imaging data on butanethiol and dodecanethiol SAMs on $\mathrm{Au}(111)$ showing unexpected molecular dynamics. Adsorbate molecule domains exhibit reversible and collective processes resulting in $\sqrt{3} \times \sqrt{ } 3 R 30^{\circ} \Leftrightarrow c(4 \times 2)$ transitions which can be observed in a range of $\sim 60 \mathrm{~s}$ at room temperature. It is also shown that the $c(4 \times 2)$ superlattice may arise from the different contrast of the $\mathrm{S}$ heads displaced from hollow to bridge substrate sites. Transitions from the $p(n \times 1)$ superlattice to the $\sqrt{ } 3 \times \sqrt{ } 3 R 30^{\circ}$ lattice were also imaged in real time.

STM imaging was performed with Nanoscope III STM equipment (Digital Instruments, Santa Barbara, CA) utilizing commercial $\mathrm{Pt}$-Ir tips, at room temperature, under ex situ conditions for butanethiol, and in situ conditions for dodecanethiol, using a Teflon cell for liquids. Au films evaporated on glass (250 nm thick Au layer onto "Robax" glass, AF Berliner glass KG, Germany) with a $2 \mathrm{~nm}$ thick chromium undercoating for better adhesion to the glass surface were used as substrates. As observed by STM and atomic force microscopy (AFM), these $\mathrm{Au}$ substrates exhibit atomically smooth (111) terraces. ${ }^{14}$

Butanethiol films were formed by dipping the Au substrate in a $50 \mu \mathrm{M}$ ethanolic solution of butanethiol for $24 \mathrm{~h}$. Then, the thiol-covered substrate was rinsed with ethanol and immediately afterwards a chosen domain was ex situ sequentially STM imaged. Dodecanethiol films were produced keeping the substrate immersed in pure thiol for $3 \mathrm{~h}$ before the in situ sequential imaging was started.

Typical tunneling resistance of about $4.5 \mathrm{G} \Omega(0.2 \mathrm{nA}$, $0.9 \mathrm{~V})$ and $10 \mathrm{G} \Omega(0.15-0.25 \mathrm{nA}, 1.5-2.5 \mathrm{~V})$ were used for butanethiol and dodecanethiol, respectively. In these ranges of operation conditions no dependence of the STM image on tunneling voltage was found.

Sequential STM images $(1 \mathrm{~V}, 150 \mathrm{pA})$ of a butanethiol 

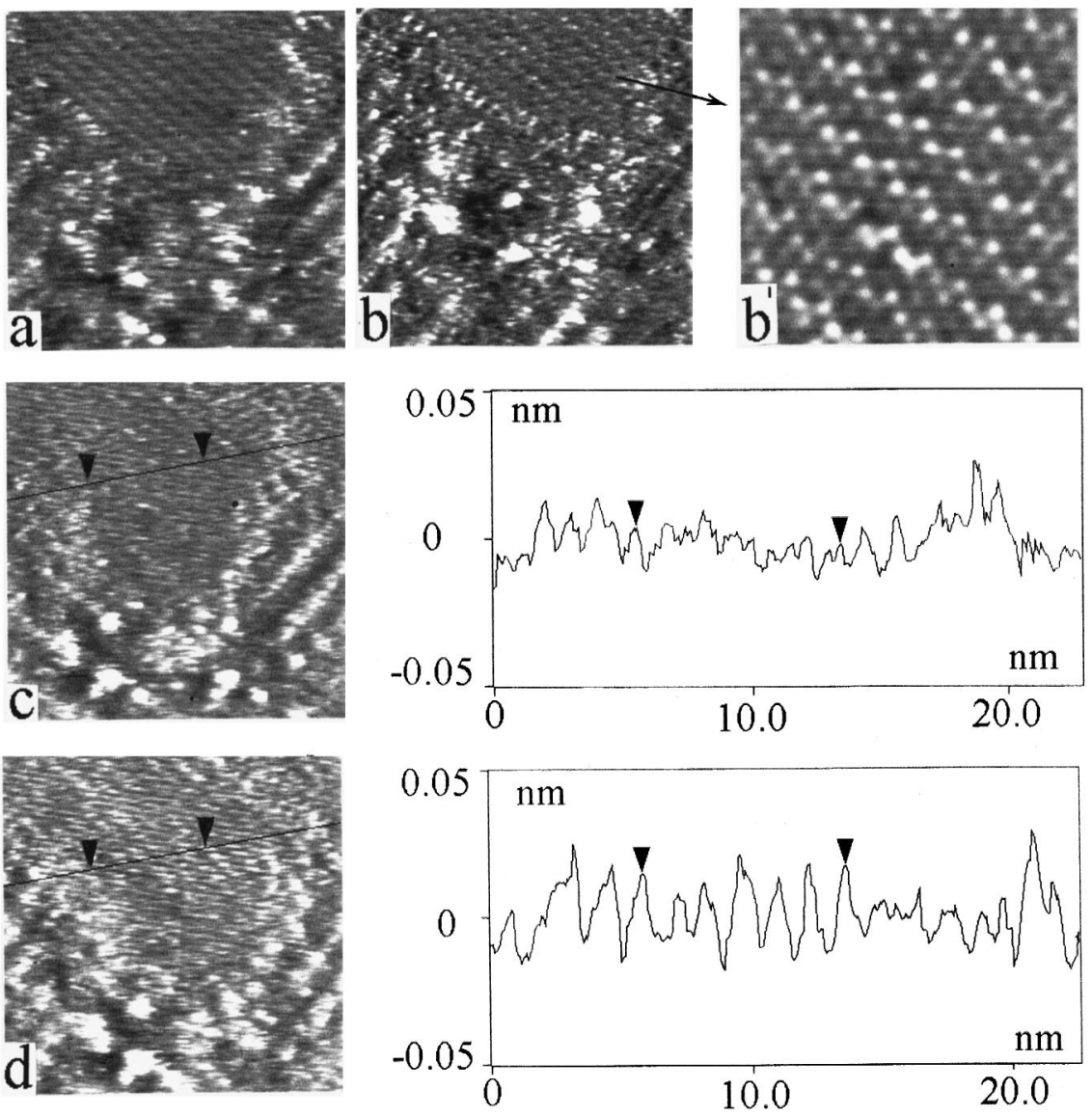

FIG. 1. Ex situ STM images (22.4 $\times 22.4 \mathrm{~nm}^{2}$ top view) of adsorbed butanethiol domains on $\mathrm{Au}(111)$ terraces. The evolution of a domain at the upper part of STM images can be seen. (a) $t=0$; (b) $t=280 \mathrm{~s}$; (c) $t$ $=420 \mathrm{~s}$; (d) $t=427 \mathrm{~s}$; (e) $t=461 \mathrm{~s}$. Cross sections of the images (c) and (d) are also shown. The latter shows the formation of rows corresponding to the $c(4 \times 2)$ superlattice. Highresolution ex situ STM images (6.67 $\times 6.67 \mathrm{~nm}^{2}$ top view) $\left(\mathrm{b}^{\prime}\right)$ and $\left(\mathrm{e}^{\prime}\right)$ of domain I and $\left(5 \times 5 \mathrm{~nm}^{2}\right.$ top view) $\left(\mathrm{e}^{\prime \prime}\right)$ of domain II are shown. Scanning was interrupted for $140 \mathrm{~s}$ between the two sets of STM images (a) and (b) and $(\mathrm{c})-(\mathrm{e})$.
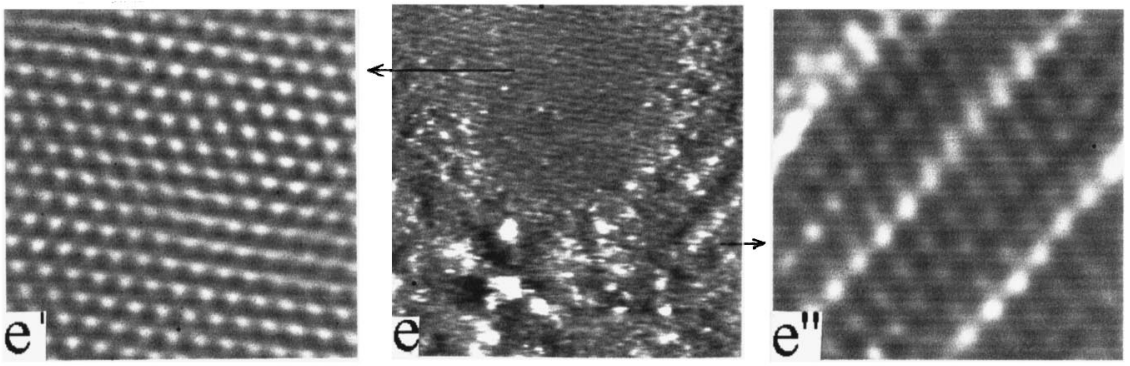

SAM on $\mathrm{Au}(111)$, prepared by adsorption from a diluted ethanolic solution (Fig. 1), show domain $I$, at the upper part of the image, revealing rows separated by $1.0 \mathrm{~nm}$, and domain $I I$, which covers the rest of the image, exhibiting rows separated by $2.0 \mathrm{~nm}$ together with randomly distributed monoatomic deep holes.

The topography of domain $I$ remains stable after imaging for $280 \mathrm{~s}$, as has already been reported, ${ }^{15,16}$ but the resolution of rows gradually increases from $t=0$ [Fig. 1(a)] to $t$ $=280 \mathrm{~s}$ [Fig. 1(b)]. High-resolution STM images of this domain [Fig. $1\left(\mathrm{~b}^{\prime}\right)$ ] reveal the presence of the $c(4 \times 2)$ lattice. To discriminate whether this structure results from tipadsorbate interaction as earlier suggested,${ }^{13}$ the experiment was continued using the protocol described in Ref. 17, displacing the tip to a region $100 \mathrm{~nm}$ away from the original site, holding it there for $2 \mathrm{~min}$, and subsequently, returning it to the initially imaged region. The immediately taken image of this region shows at the central part the disappearance of adsorbate rows [Fig. 1(c), and cross section]. These rows, however, tend to reappear a few seconds later [Fig. 1(d)], and cross section), and finally disappear almost completely [Fig. 1(e)] in favor of the well-known $\sqrt{3} \times \sqrt{3} R 30^{\circ}$ lattice with a $0.5 \mathrm{~nm}$ distance between the spots [Fig. 1( $\left.\left(\mathrm{e}^{\prime}\right)\right]$. Therefore, this experiment shows the successive transitions $c(4$ $\times 2) \Rightarrow \sqrt{ } 3 \times \sqrt{ } 3 \quad R 30^{\circ} \rightarrow c(4 \times 2) \rightarrow \sqrt{ } 3 \times \sqrt{ } 3 \quad R 30^{\circ}$ in the adsorbate domain. It is clear that for butanethiol adsorbate the $c(4 \times 2)$ superlattice under scanning is less stable than expected according to previously reported data. ${ }^{13,15,16}$

Two different types of spots emerge from a detailed analysis of the $c(4 \times 2)$ supperlattice [Fig. 2(a)]. From the cross section [Fig. 2(b)] a $0.45 \mathrm{~nm}$ separation between bright and gray neighbor spots was determined rather than the 0.5 $\mathrm{nm}$ separation expected for the $\sqrt{3} \times \sqrt{ } 3 R 30^{\circ}$ lattice. The difference of $0.05 \mathrm{~nm}$ is consistent with the displacement of the thiol molecule from a hollow to a bridge site. The adsorption of the S head at different sites would result in STM images with different spot contrast.

Similar images have been obtained for octanethiol on $\mathrm{Au}(111),{ }^{17}$ but as no particular attention to the bright-to-gray spot distance has been paid there, it has been concluded that 


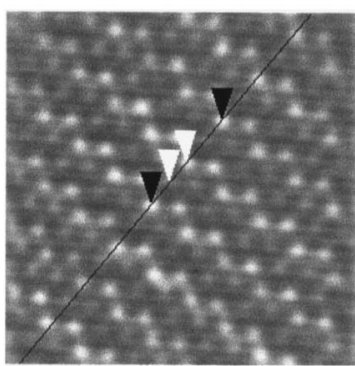

a
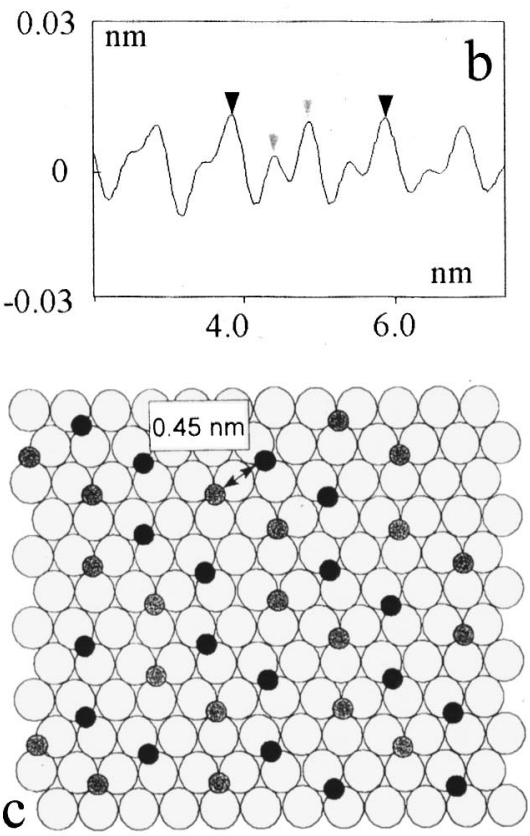

FIG. 2. A STM image $\left(6.67 \times 6.67 \mathrm{~nm}^{2}\right)$ (a) and cross section (b) showing the position of S-thiol heads (see arrows in the cross section) in the $c(4$ $\times 2$ ) lattice. Note the $1.0 \mathrm{~nm}$ distance between alternating rows and the 0.45 $\mathrm{nm}$ distance between adjacent rows. Scheme (c) showing the model proposed to explain the $c(4 \times 2)$ lattice. Alternating rows of $\mathrm{S}$ heads at hollow (gray circles) and bridge (black circles) sites are consistent with the distances shown in the cross section.

the $\mathrm{S}$ heads are all at equivalent sites and the difference in contrast is due to some variation in molecular orientation. However, if one admits that STM images the S head of the thiol molecules, ${ }^{18,19}$ a different conclusion can be drawn. In this way, our results are consistent with $\mathrm{S}$ heads located at distinguishable sites on the $\mathrm{Au}(111)$ surface as shown in Fig. 2(c). According to our conclusion, the adsorbate layer shown in Fig. 1 involves distinguishable domains with thiol molecules located at hollow sites forming the $\sqrt{ } 3 \times \sqrt{ } 3 R 30^{\circ}$ lattice, and domains with thiol molecules placed at hollow and bridge sites forming the $c(4 \times 2)$ superlattice. This conclusion, which has been recently suggested from sum-frequency generation spectral data, ${ }^{20}$ is consistent with the fact that no dependence of the structure of these lattice domains with tunneling voltage was observed.

Bright spots forming rows separated by $2.0 \mathrm{~nm}$ are visible in domain $I I$, for at least $460 \mathrm{~s}$ [Figs. 1(a)-(e)], exhibiting a $p(4 \times 1)$ superlattice [Fig. $\left.1\left(\mathrm{e}^{\prime \prime}\right)\right]$. In fact, the $p(3$ $\times 1)$ superlattice has recently been observed as a transient structure during the $\sqrt{ } 3 \times \sqrt{ } 3 R 30^{\circ} \Rightarrow c(4 \times 2)$ transition. ${ }^{13}$

The in situ STM images of SAMs produced by immers-
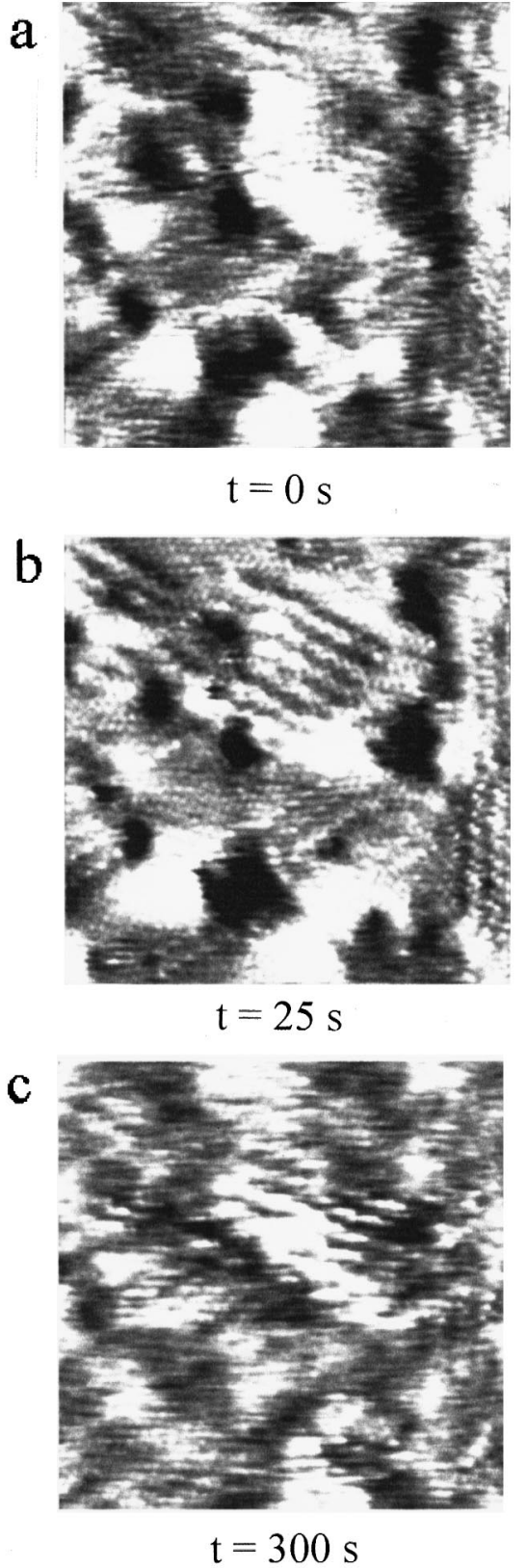

FIG. 3. Sequential (a)-(c) in situ STM images $\left(20 \times 20 \mathrm{~nm}^{2}\right.$ top view) of adsorbed dodecanethiol domains on $\mathrm{Au}(111)$ terraces. A detailed inspection of the images shows the $\sqrt{ } 3 \times \sqrt{ } 3 R 30^{\circ} \Leftrightarrow c(4 \times 2)$ transitions at different places.

ing the $\mathrm{Au}(111)$ specimen in dodecanethiol also consist of differently structured adsorbate domains and holes [Figs. 3(a)-3(c)]. In this case, the size of the monoatomic deep holes also changes from one image to the other. The dynamics of dodecanethiol SAM domains followed by in situ STM comprises the same type of $\sqrt{3} \times \sqrt{3} \quad R 30^{\circ} \Leftrightarrow c(4 \times 2)$ transitions [Figs. 3(a)-3(c)] described above for butanethiol SAMs. Accordingly, it appears that these transitions are independent of the thiol alkyl chain length and adsorbate preparation conditions. For the dodecanethiol SAMs, transitions from the $p(6 \times 1)$ superlattice to the $\sqrt{ } 3 \times \sqrt{ } 3 R 30^{\circ}$ lattice occur within a few minutes [Figs. 4(a) and 4(b)]. In contrast to previous data, ${ }^{17}$ molecular resolution at inter-row spacing of the $p(6 \times 1)$ lattice [Fig. 4(a)] indicates the same 

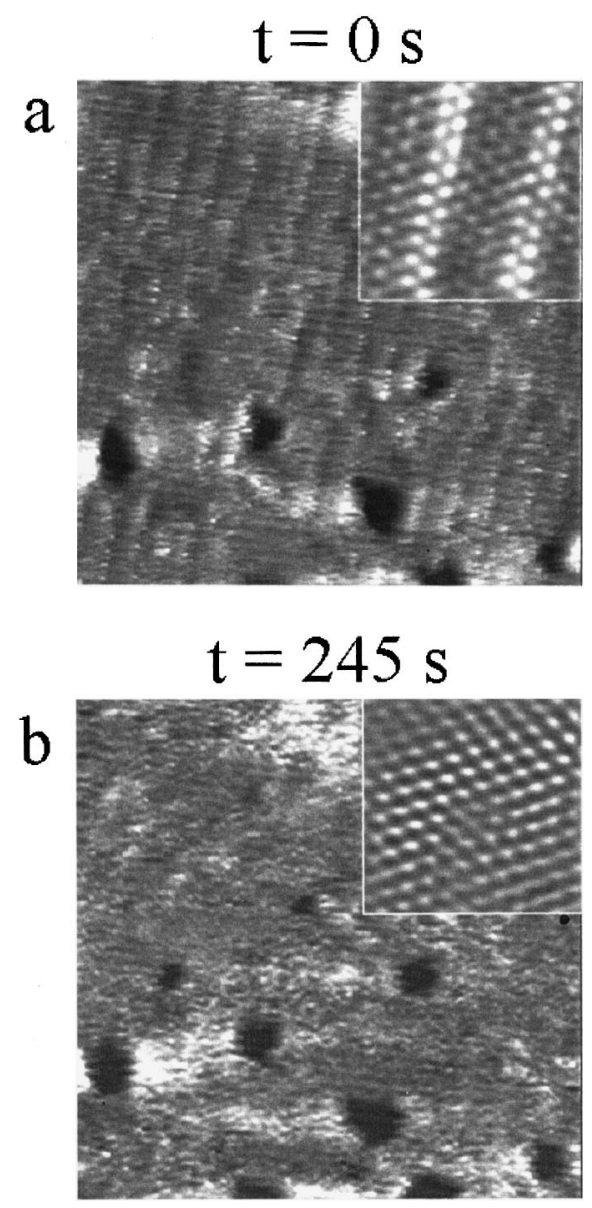

FIG. 4. Sequential (a) and (b) in situ STM images $\left(40 \times 40 \mathrm{~nm}^{2}\right.$, top view) of dodecanethiol domains on $\mathrm{Au}(111)$ terraces. The $p(6 \times 1)$ superlattice (a), and the $\sqrt{ } 3 \times \sqrt{ } 3 R 30^{\circ}$ lattice (b) are shown (insets). Note that in (b) the stripes of the $p(6 \times 1)$ superlattice have disappeared.

packing density found in the $c(4 \times 2)$ and $\sqrt{ } 3 \times \sqrt{ } 3 R 30^{\circ}$ lattice. Bright rows at similar distances have been assigned to thiol molecules parallel to the substrate surface at the early stages of monolayer formation. ${ }^{18}$ In our case, this explanation fails to account for the hexagonal arrangement of molecules at inter-row spacing shown in Fig. 4(a). Therefore, for both thiol adsorbates the $p(n \times 1)$ superlattice would result from rows of thiol molecules being adsorbed on hep rather than on fcc sites of $\mathrm{Au}(111){ }^{21}$

The reversible $\sqrt{3} \times \sqrt{ } 3 R 30^{\circ} \Leftrightarrow c(4 \times 2)$ transition at room temperature described above, which is detected in the range of $10 \mathrm{~s}$ or thereabouts, would involve the collective motion of a number of thiol molecules, as would be expected for oscillating systems. ${ }^{22}$

The adsorption energy change $(\Delta E)$ related to the displacement of the alkanethiol molecule from a fcc to a bridge site on $\mathrm{Au}(111)$ results in $\Delta E \approx 4.2 \mathrm{~kJ} / \mathrm{mol}$, whereas the adsorption energy change from either a hcp to a bridge site or a hcp to a fcc site is $\Delta E \approx 4.2-8.4 \mathrm{~kJ} / \mathrm{mol}^{23}$ These values of $\Delta E$ are 2-4 times greater than the thermal fluctuation energy of adsorbates at $298 \mathrm{~K}$. Accordingly, other possible energy contributions for promoting the adlayer structural transitions have to be discussed.

Typical changes in size of randomly distributed holes accompanying alkanethiol adlayer lattice transitions are of about $100 \mathrm{~nm}^{2}$ in surface area. Then, taking for the surface tension of $\mathrm{Au}(111)$ at $298 \mathrm{~K}$ is $\gamma \cong 1000 \mathrm{erg} / \mathrm{cm}^{2}$, a decrease of $1 \mathrm{~nm}^{2}$ in surface area would mean an energy release of about $10^{-11} \mathrm{erg}$. This energy can be either dissipated in the surroundings or used by adsorbed molecules to change the position from hollow to bridge sites promoting the $(\sqrt{ } 3$ $\times \sqrt{ } 3) \quad R 30^{\circ} \Rightarrow c(4 \times 2)$ structural transition. Hence, the amount of energy released in decreasing in hole area becomes sufficient to assist the change in the position of nearly 200 adsorbed molecules occupying a $13 \times 13 \mathrm{~nm}^{2}$ domain. Otherwise, when thiol molecules are involved in the reverse process, i.e., $c(4 \times 2) \Rightarrow(\sqrt{3} \times \sqrt{ } 3) R 30^{\circ}$, moving adsorbate molecules from bridge to hollow sites, energy is provided to grow small holes. Then, the repetitive process would lead to a fluctuating quasi two-dimensional (2D) system with a small damping.

In conclusion, the transitions $p(6 \times 1) \Rightarrow \sqrt{ } 3$ $\times \sqrt{ } 3 R 30^{\circ} \Leftrightarrow c(4 \times 2)$ in the adlayer structure have been demonstrated. The reversible transition could be originated in the alternating displacements of thiol molecules from hollow to bridge and from bridge to hollow positions coupled with the change in size of monoatomic deep holes of the $\mathrm{Au}(111)$ surface.

Grants from the Consejo Nacional de Investigaciones Científicas y Técnicas (CONICET) (PIP 014/97 and PIA 7283/97) and Comisión de Investigaciones Científicas (CIC) of Pcia. de Buenos Aires are acknowledged.

${ }^{1}$ L. H. Dubois and R. G. Nuzzo, Annu. Rev. Phys. Chem. 43, 437 (1992).

${ }^{2}$ N. Camillone III, C. E. Chidsey, G.-Y. Liu, and G. Scoles, J. Chem. Phys. 98, 3503 (1993).

${ }^{3}$ A. Ulman, An Introduction to Ultrathin Organic Films, from Langmuir Blodgett to Self-Assembly (Academic, San Diego, CA, 1991).

${ }^{4}$ N. Camillone III, C. E. Chidsey, G.-Y. Liu, T. M. Putvinski, and G. Scoles, J. Chem. Phys. 94, 8493 (1991).

${ }^{5}$ L. H. Dubois, B. R. Zegarski, and R. G. Nuzzo, J. Chem. Phys. 98, 678 (1993).

${ }^{6}$ R. G. Nuzzo, L. H. Dubois, and D. L. Allara, J. Am. Chem. Soc. 112, 558 (1990).

${ }^{7}$ H. Sellers, Surf. Sci. 294, 99 (1993).

${ }^{8}$ M. G. Samant, C. A. Brown, and J. G. Gordon, Langmuir 7, 437 (1991).

${ }^{9}$ D. Anselmetti, A. Baratoff, H. J. Guntherodt, E. Delamarche, B. Michel, Ch. Gerber, H. Kang, H. Wolf, and H. Ringsdorf, Europhys. Lett. 27, 365 (1994).

${ }^{10}$ N. Camillone III, C. E. D. Chidsey, P. Eisenberger, P. Fenter, J. Li, K. S. Liang, G. Y. Liu, and G. Scoles, J. Chem. Phys. 99, 744 (1993).

${ }^{11}$ P. Fenter, A. Eberhardt, and P. Eisenberger, Science 266, 1216 (1994).

${ }^{12}$ T. Ishida, S. Yamamoto, W. Mizutani, M. Motomatsu, H. Tokumoto, H. Hokari, H. Azehara, and M. Fujihira, Langmuir 13, 3261 (1997).

${ }^{13}$ I. Touzov and C. B. Gorman, J. Phys. Chem. B 101, 5263 (1997).

${ }^{14}$ F. Terán Arce, M. E. Vela, R. C. Salvarezza, and A. J. Arvia, Surf. Rev. Lett. 4, 637 (1997).

${ }^{15}$ G. E. Poirier and M. J. Tarlov, Langmuir 10, 2853 (1994)

${ }^{16}$ E. Delamarche, B. Michel, and Ch. Gerber, Langmuir 10, 2869 (1994).

${ }^{17}$ G. E. Poirier, M. J. Tarlov, and H. E. Rushmeir, Langmuir 10, 3383 (1994).

${ }^{18}$ G. E. Poirier and E. D. Pylant, Science 272, 1145 (1996).

${ }^{19}$ A. Karpfen, J. Chem. Phys. 75, 238 (1981).

${ }^{20}$ M. S. Yaganeh, S. M. Dougal, R. S. Polizzotti, and P. Rabinowitz, Phys. Rev. Lett. 74, 1811 (1995).

${ }^{21}$ J. Kang and P. A. Rowntree, Langmuir 12, 2813 (1996).

${ }^{22}$ G. Nicolis and I. Prigogine, Self-Organization in Nonequilibrium Systems (Wiley-Interscience, New York, 1977).

${ }^{23}$ K. M. Beardmore, J. D. Kress, A. R. Bishop, and N. Gronbech-Jensen, Synth. Met. 84, 317 (1997). 